\title{
¿Es posible obtener un título de obtentor sobre una mutación vegetal espontánea en el Ecuador?
}

Is it possible to get a breeder on a spontaneous vegetable mutation in Ecuador?

\author{
María Fernanda Valdospinos Rodríguez \\ Investigadora independiente
}

\begin{abstract}
Resumen
El presente artículo busca determinar si los obtentores vegetales pueden ser titulares de variedades esencialmente derivadas que se han obtenido mediante métodos en los que no existe intervención humana. Por consiguiente, el análisis consiste en establecer si la puesta a punto o mejora vegetal que prevén las normas se cumple en estos casos. Con esta problemática, cabe preguntarse si se pueden proteger, bajo la legislación local, variedades esencialmente derivadas en las que no ha existido intervención del ser humano.

La legislación ecuatoriana no incluye este concepto expresamente y el único artículo en el que se hace referencia es el 467 , sobre las limitaciones del obtentor. Lo que no permite determinar qué ocurre con esta categoría en nuestra legislación. Sin embargo, la Decisión 345 agrega este concepto y lo amplía en varios apartados que serán ampliados a continuación.
\end{abstract}

\section{Palabras Clave}

Obtentores vegetales / Variedades esencialmente derivadas / Métodos de obtención / Selección natural.

\section{Summary}

This article seeks to clarify if plant breeders are also entitled to essentially derived rights on varieties that has been obtain by methods in which there was no human intervention. Therefore, the analysis consists in determining if the required plant breeding is present in this case. In this regard, the question to be answer is whether or not is possible to protect under the local law essentially derived varieties in which there has been no human intervention.

Ecuadorian legislation does not include this concept and in the only article in which reference is in article 467, about the limitations of the breeder. What cannot determine what happens to this category in our legislation. However, Decision 345 adds this concept and expands it in some sections that will be study below.

\section{Keywords}

Plant Breeders / Essentially derived varieties / Breeding methods / Natural selection.

\section{Introducción}

Ecuador es suscriptor del Acta del Convenio Internacional para la Protección de las Obtenciones Vegetales (en adelante Convenio de la UPOV) de 1978. Además, en su calidad de Miembro de la Comunidad Andina, está sujeto a la Decisión N³45 sobre el Régimen Común de Protección a los Derechos de los Obtentores de Variedades Vegetales de la Comunidad Andina (en adelante Decisión $\left.N^{\circ} 345\right)$ así como, en la legislación local, al Código Orgánico de la Economía Social de los Conocimientos, Creatividad e Innovación (en adelante COESC) de 2016. Estos cuerpos normativos difieren en conceptos, dejando en ambigüedad algunos temas. En el caso que nos 
atañe, el COESC simplemente trae el concepto, sin incorporar aclaraciones; por su parte, la Decisión No345 agrega el concepto, mientras que la UPOV de 1978 no lo incluye.

Como es de conocimiento general, la legislación comunitaria prevalece sobre la legislación nacional, y dado que nuestra normativa local actual fue modificada simplemente se agrega el concepto a breves rasgos, sin dejar claro en qué casos cabe la posibilidad de registrar una variedad esencialmente derivada. Dejando de lado la ambigüedad que existe sobre el tema, se puede identificar que la problemática está dada porque al introducir el concepto de variedad esencialmente derivada, se hace referencia al Convenio de la UPOV de 1991, en la que se definen algunos métodos de obtención, dentro de los cuales se encuentra la selección de mutantes naturales. Aunque, en el COESC y en la Decisión No345 no existe tal apartado, es incierto si en el país están permitidos o excluidos este tipo de métodos de obtención vegetal.

En la práctica, en Ecuador, hasta el momento, no se han recibido solicitudes de obtentor vegetal sobre una variedad esencialmente derivada, pues, en primer lugar, no existe una metodología para solicitar tal certificado; $y$, en segundo lugar, tampoco existe un organismo técnico encargado de explorar si el certificado le corresponde a una variedad principal o a una variedad esencialmente derivada. Según información del Instituto Ecuatoriano de Propiedad Intelectual (IEPI) y la Ley de Propiedad Intelectual que estaba vigente hasta el año 2016, la obtención del certificado se basa en completar un formulario que no analiza la parte técnica.

La existencia de esta apertura para registrar cualquier tipo de obtención, sin limitar los métodos y, específicamente, la selección de mutantes naturales, permitiría la protección de hallazgos o descubrimientos, los cuales, por la estructura del sistema, se encuentran totalmente excluidos. Pese a lo planteado anteriormente, se debe señalar que el Convenio del UPOV de 1991, de donde se tomó esta figura de variedades esencialmente derivadas, permite tal hecho, ampliando los derechos del obtentor vegetal a una derivación, incluso de una variedad que se ha dado de forma espontánea.

\section{Definiciones}

Para fines del análisis, definimos los siguientes términos relacionados con el ámbito de la obtención vegetal:

- Variedades Vegetales: conjunto de individuos botánicos cultivados que se distinguen por determinados caracteres morfológicos, fisiológicos, citológicos, químicos, que se pueden perpetuar por reproducción, multiplicación o propagación (Decisión No345, 1993).

- Obtenciones Vegetales: son variedades en las cuales el ser humano ha intervenido desarrollando, manipulando o aplicando una técnica.

- Puesta a punto (crear): es uno de los requisitos esenciales para ser declarado obtentor vegetal, y se define como la aplicación de alguna técnica de fitomejoramiento.

- Obtentor Vegetal: es el sujeto que debe realizar un trabajo de investigación y haber obtenido una variedad vegetal mediante un proceso de mejoramiento. El obtentor puede exigir que se le otorguen los derechos exclusivos sobre su variedad, si esta reúne las características exigidas por la ley, con especial énfasis en el mejoramiento vegetal; así como la novedad, homogeneidad, estabilidad y distinguibilidad.

- Variedades Esencialmente Derivadas: aquella que se origine de esta o de una variedad 
que a su vez se desprenda principalmente de la primera, conservando las expresiones de los caracteres esenciales que resulten del genotipo o de la combinación de genotipos de la variedad original, $y$, aun, si se puede distinguir claramente de la variedad inicial, concuerda con esta en la expresión de los caracteres esenciales resultantes del genotipo o de la combinación de genotipos de la primera variedad, salvo por lo que respecta a las diferencias resultantes del proceso de derivación (Patińo, 1998, p. 41).

- Métodos de Obtención: la hibridación es la construcción artificial de ácidos nucleicos bicatenarios a partir de dos monocatenarios usando la complementariedad de bases. Se trata, por tanto, de un proceso de unión de dos cadenas complementarias de $\mathrm{ADN}, \mathrm{ARN}$ o de ADN y ARN para formar una molécula de ácido nucleico de doble cadena (Fernández Ruiz, 2016). Además, el Artículo 14.5 c) del Convenio de la UPOV de 1991 ejemplifica como métodos la selección de un mutante natural o inducido o de una variante somaclonal, selección de un individuo variante entre las plantas de la variedad inicial, retrocuzamientos o transformaciones por ingeniería genética.

\section{Normas aplicables}

Las siguientes fuentes normativas debe tomarse en cuenta para el análisis:

- Convenio de la UPOV 1978

- Convenio de la UPOV 1991

- Decisión No345 de la Comisión de la Comunidad Andina

- Código Orgánico de la Economía Social de los Conocimientos, Creatividad e Innovación (COESC)

\section{La selección de mutantes naturales como método de obtención}

La selección natural es una herramienta fundamental en la mejora de plantas, es la clave de éxito del mejorador vegetal, y reside en poder reconocer tipos superiores en un limitado o amplio rango de variabilidad (Idareta Olagüe, 2004, p. 3). El artículo 14.5 c) de la UPOV de 1991, incluye como método de fitomejoramiento, la selección de un mutante natural.

El concepto de selección natural, se puede definir como un

proceso de mejora genética que la naturaleza realiza a lo largo de numerosas generaciones. Este principio ya fue enunciado por Charles Darwin en 1859 mediante su teoría de la evolución de las especies, por lo cual la selección natural es una consecuencia de la lucha de los seres vivos por la propia existencia, lo que da lugar a la supervivencia de aquellos más aptos; estas características son así transmitidas a los descendientes, que obtienen mejoras genéticas para enfrentarse a la vida en condiciones más favorables (INFO AGRO, 2014).

Asimismo, según Fernando Muñoz define, la selección natural o conservadora “[c]onsiste en escoger, entre los individuos de una misma especie, aquellos de mejor aspecto morfológico, floración precoz, resistencia a las plagas, etc., los que den mejor rendimiento y calidad de un principio activo determinado" (Muńoz, 2002, p. 25).

La selección no es resultado de la adaptación de un individuo, sino de la existencia de variantes dentro de la población que tengan mayor poder de supervivencia y permitan la continuidad de la especia toda. Por ejemplo, la aplicación de un herbicida X destruye la gran mayoría de malezas, pero existe una variante entre la especie de mayor resistencia, la cual podrá sobrevivir y propagarse rápidamente ocupando los espacios liberados por las malezas destruidas (Consejo Argentino para la Información y el Desarrollo de la Biotecnología). 
La selección natural también puede ser una actividad llevada a cabo por el ser humano, esta actividad se conoce como selección artificial. El ser humano acelera el proceso de mejora y selección natural, con la intención de obtener resultados que le beneficien. La actividad consiste en seleccionar aquellas plantas deseables por determinadas características y desechar las demás. La selección artificial consiste en elegir los individuos progenitores cuyo fenotipo es más favorable. y el proceso de mejora consiste en seleccionar aquellas que dispongan de caracteres deseables en el mayor grado posible desechando los de grado menor, para posteriormente repetir la operación durante varias generaciones hasta alcanzar las expectativas deseadas (Cervantes).

Aun en la selección natural-artificial en la que el ser humano ha intervenido con el fin de acelerar el proceso, se trata de un método, cuya intervención es una mera selección de las plantas que han sido más adaptables, por lo que no se puede afirmar que exista una aplicación de una técnica o mejoramiento vegetal.

La puesta a punto que se ha planteado como requisito esencial para el otorgamiento de título de obtentor vegetal, se refiere a que, si bien el ser humano pudo haber descubierto la variedad, se requiere que haya un proceso de

reproducción o multiplicación y evaluación, aunque estos es lo que el Acta de la UPOV de 1991 describe como puesta a punta, existe un grupo numeroso que considera que "solo se cumple con el criterio de puesta a punto si la propia planta descubierta se modifica luego de alguna manera y que la reproducción o la multiplicación de la planta sin modificaciones no constituye una puesta a punto" (UPOV, 2000b).

En lo que respecta al método, no se puede considerar que la reproducción o la multiplicación sean una mejora vegetal, pues únicamente se trata de una reproducción de la variedad, que es resultado de la naturaleza. En este método se debe exigir que la planta descubierta tenga reproducción sexual y que se efectúe la selección en la progenie para tener una prueba de la puesta a punto. Tanto es así que la norma indica que será obtentor la persona que crea una variedad, es decir, la persona que utiliza métodos y técnicas de mejoramiento vegetal, que generalmente se debe entender como el uso de ingeniería genética.

Las variedades vegetales son parte de la propiedad intelectual y por eso deben cumplir con todos principios de este sistema. La propiedad intelectual en términos generales, es "toda creación del intelecto humano" (Canaval, 2008, p. 8), entonces el obtentor debe demostrar que ha realizado una creación, lo que en el ámbito de las variedades vegetales significa utilizar métodos y técnicas de mejoramiento vegetal.

La selección natural incluso puede resultar peligrosa para la biodiversidad, pues según Miguel Ángel Cervantes Flores, Profesor titular del Centro F.P. Campomar "los distintos centros de investigación de semillas, se desplazan a zonas salvajes, sobre todo de América Central y América del Sur, y escogen aquellas plantas que mejor se han adaptado a las condiciones adversas, para seleccionarlas por su resistencia” (Cervantes). Es así que se podría seleccionar variedades nativas, y dado que no existe un inventario ni regulación sobre los métodos, podrían incluso llegar a ser sujetas de protección.

El título de obtentor debe proteger los derechos de éste de forma correlativa al esfuerzo que se ha invertido, dado que en la selección natural no existe una gran inversión económica, ni de tiempo de investigación. Por lo que podría resultar injusto para aquellos breeders que han dedicado mucho tiempo y dinero a la obtención de una variedad.

Para CIOPORA los mutantes naturales también resultan problemáticos, en especial para las variedades ornamentales y frutales de multiplicación vegetativa. Consideran que los 
mutantes presentan varias ventajas comerciales al descubridor: no precisan mucha labor de descubrimiento y puesta a punto, su proceso de evaluación es breve, se benefician de la variedad ya conocida y son fáciles de introducir en el mercado. Por otro lado, se requiere una inversión reducida, los mutantes de una variedad innovadora inicial pueden usurpar esta una gran cuota de mercado, y la rentabilidad que para su inversión consiga el obtentor de la variedad innovadora inicial no será la misma si esta comparte su nicho de mercado con mutantes de bajo costo (Krieger, 2013). Si se analiza lo expuesto por CIOPORA, se puede identificar incluso que es contrario a las justificaciones de la UPOV permitir la protección de variedades obtenidas mediante selección natural, pues incluso podría existir una lesión a los beneficios de un obtentor. Es así que, por principio, todos los obtentores deberían invertir una cantidad equivalente de dinero y tiempo, caso contrario podría tratarse de una competencia injusta.

En conclusión, es indudable que la selección natural es un inicio muy importante para lograr el fitomejoramiento de plantas. Por concepto, la selección natural es un hecho de la naturaleza que intenta la sobrevivencia/existencia de una especie ante un sinnúmero de adversidades, en esta actividad se puede verificar que el ser humano no ha hecho una inversión significativa, por lo menos en este método puro, simplemente ha tomado la variedad de la naturaleza y la ha propagado, sin aplicar ninguna técnica. Existen técnicas de fitomejoramiento que demuestran una real aplicación de una técnica, en la que se puede verificar que el ser humano ha invertido tiempo y conocimientos en lograr la obtención de esta variedad esencialmente derivada. Permitir la existencia de la selección natural como un método de fitomejoramiento podría generar dańos a los principios de propiedad intelectual y esencialmente a los derechos de obtentores, porque se estaría permitiendo la protección de descubrimientos. La selección natural de mutantes es una práctica contraria a todos los principios de la propiedad intelectual.

\section{La puesta a punto como requisito esencial de las variedades vegetales}

\begin{tabular}{|l|l|}
\hline NORMA & ARTÍCULO \\
\hline Convenio de UPOV 1978 & No presta una definición. \\
\hline Decisión 345 & $\begin{array}{l}\text { Artículo 4.- } \\
\text { Los Países Miembros otorgarán certificados de obtentor a } \\
\text { las personas que hayan creado variedades vegetales, cuando } \\
\text { éstas sean nuevas, homogéneas, distinguibles y estables y } \\
\text { se le hubiese asignado una denominación que constituya su } \\
\text { designación genérica. } \\
\text { Para los efectos de la presente Decisión, entiéndase por crear, } \\
\text { la obtención de una nueva variedad mediante la aplicación de } \\
\text { conocimientos científicos al mejoramiento heredable de las } \\
\text { plantas (el énfasis es mío). }\end{array}$ \\
\hline $\begin{array}{l}\text { Código Orgánico de la Economía Social } \\
\text { de los Conocimientos e Innovación }\end{array}$ & $\begin{array}{l}\text { Artículo 448.- A estos efectos, se entiende por crear la obtención } \\
\text { de una nueva variedad mediante la aplicación de conocimientos } \\
\text { científicos al mejoramiento heredable de las plantas (el énfasis } \\
\text { es mío). }\end{array}$ \\
\hline Convenio de la UPOV 1991 & $\begin{array}{l}\text { Artículo 1.- Se entenderá por obtentor } \\
\text { iv) - la persona que haya creado o descubierto y puesto a punto } \\
\text { una variedad (el énfasis es mío). }\end{array}$ \\
\hline
\end{tabular}

La puesta a punto es un requisito del Convenio de la UPOV de 1991, por lo que no es relevante, en esos términos, en nuestro país. Sin embargo, las normas que sí son relevantes también tocan 
ese tema, aunque con otro nombre, pero se refieren al mismo requisito, la Decisión No345 apunta a que se requiere crear de la mano con el requisito del COESC.

El análisis de la norma se hará conforme al Convenio de la UPOV de 1991 porque parece aclarar de mejor manera la puesta a punto de las variedades. Para que un obtentor se considere como tal, el Artículo 1 del Convenio de la UPOV de 1991 establece el concepto de obtentor "la persona que haya creado o descubierto y puesto a punto una variedad", lo que significa que el simple descubrimiento ${ }^{1}$ o hallazgo no daría lugar a la concesión de un derecho de obtentor. De esta forma, crear se considera como un requisito previo para la concesión de un derecho de obtentor, por esta razón no se otorgará tal protección a una persona que haya descubierto y reproducido sin cambios.

Según la definición de la UPOV, la puesta a punto es el proceso de reproducción o multiplicación y evaluación. La multiplicación vegetativa, también conocida como reproducción asexual, "es la que se produce sin la unión de los núcleos de las células sexuales o gametos, a partir de otras células del individuo adulto ya desarrollado, de tal manera que el individuo resultante es desde el punto vista genético idéntico al parental" (UNNE, 2015), en cambio, la reproducción vegetativa "tiene lugar por fragmentación del individuo adulto o a partir de estructuras asexuales especiales" (UNNE, 2015). Esto significa que no existe una verdadera modificación genética ni fenotípica, sino que se trata de una multiplicación en cantidad de la variedad, y, por principio, eso no es una actividad sujeta a protección.

En los debates de la UPOV, un grupo de panelistas argumentó que una variedad no cumple con el requisito de mejora vegetal, si la planta descubierta se modifica mediante la reproducción o multiplicación de la planta sin más modificaciones, es decir, no constituye una puesta a punto. Esto exigiría que la planta descubierta se reprodujera en forma sexual y que se efectuara una selección en la progenie para una prueba de la puesta a punto. Los opositores a este enfoque consideran que ese argumento no es correcto, pues la selección de la progenie constituiría un fitomejoramiento, además agregan que aceptar la primera postura denegaría la protección a las mutaciones, puesto que estas se reproducen o multiplican sin modificaciones (UPOV, 2002).

En el Comité Administrativo y Jurídico de la UPOV, en la cuadragésima primera sesión del 6 de abril de 2000, la Delegación de Estados Unidos señaló que era inadecuado definir el término puesta a punto como "mejora genética". En la práctica los obtentores no podían solicitar de manera inmediata la protección simplemente tras recoger el material vegetal en la naturaleza. Después del descubrimiento de material vegetal en la naturaleza, el obtentor debía domesticar o cultivar el material seleccionado como variedad. Este proceso debería considerarse como puesta a punto (UPOV, 2000a)

Por su parte, la Delegación de Francia, en ese mismo Comité, consideró que, únicamente se cumpliría el criterio de puesta a punto, si la planta descubierta se modificaba

\footnotetext{
${ }^{1}$ Cuando se revisó el Convenio de la UPOV en 1991, pese al hecho de que el efectuar selecciones dentro de una variación preexistente pasaba por una actividad normal de los obtentores, se consideró útil incluir una definición de obtentor a fin de poner de relieve un hecho de que la versión anterior recogida en el acta de 1978 también preveía la protección de variedades que habían sido descubiertas. Sin embargo, en la Conferencia Diplomática, los delegados eran conscientes de que los descubrimientos eran muy importantes para la mejora vegetal, pero reconocieron que, en la práctica, un descubrimiento debe evaluarse y difundirse antes de poder ser explotado. Este es el motivo por el que se decidió utilizar en el Artículo 1.iv) del Acta de 1991 la noción de obtentor incluyendo a la persona que haya creado o descubierto y puesto a punto una variedad. La referencia al origen, artificial o natural, de la variación inicial que ha dado lugar a la variedad, que figura en el Artículo 6.1.a) del Acta de 1978 ya no aparece. En el Acta de 1991, el descubrimiento describe la actividad de "selección dentro de la variación natural", mientras que la "puesta a punto" describe el proceso de "reproducción o multiplicación y evaluación".
} 
posteriormente de alguna manera. Es decir, que la multiplicación, sin ninguna mejora, de una planta descubierta en la naturaleza no sería suficiente para satisfacer las condiciones del "descubrir y poner a punto". Consecuentemente, una variedad obtenida mediante un simple descubrimiento efectuado en la naturaleza no estaría en condiciones de ser protegida (UPOV, 2000a) Francia considera de esta forma que la selección natural o el descubrimiento de la naturaleza no satisfacen el criterio de "descubrir y poner a punto".

Asimismo, el representante de ASSINSEL en el Comité opinó que, si no existía alguna "mejora" en las variedades, los solicitantes no tenían derecho a la protección. Sin embargo, la mejora vegetal no podía definirse fácilmente, sólo podían detallarse los casos en los que se producía la mejora: no debían estar protegidas las variedades descubiertas en la naturaleza que no habían sufrido ninguna variación o mejora, es decir, "reproducidas por multiplicación de la misma manera”. Sin embargo, no podía considerarse puesta a punto el simple descubrimiento de una variedad dentro de una variedad existente en la naturaleza.

Continuando con las posiciones en el Comité del 2000, el Presidente de esa organización preguntó si podría protegerse una mutación existente en la naturaleza. La Delegación de Francia declaró que la respuesta dependía del modo de reproducción o multiplicación, o sea, de la necesidad de que se produjeran otras actividades de obtención después el descubrimiento. Otro punto de discusión fue determinar si una mutación existente en la naturaleza ya formaba parte de las variedades notoriamente conocidas. Sin embargo, la Delegación insistió en que no tendrían derecho a la protección las variedades de multiplicación vegetativa que eran mutaciones existentes en la naturaleza, pero podría protegerse una variedad de multiplicación vegetativa que procediera de una mutación surgida en el seno de una variedad cultivada, si satisfacía las condiciones de protección. Además, consideraron que era importante poder determinar si la variedad se lograba a través de una variación surgida en la naturaleza o una variación creada artificialmente. Por lo tanto, debía examinarse cada caso de forma individual para determinar si se trata de un caso de una variedad notoriamente conocida (UPOV, 2000a).

El Presidente del Comité Técnico señaló que existía la posibilidad de lograr obtenciones mediante material vegetal presente en la naturaleza. El proceso no sólo consiste en tomar una planta de la naturaleza, se requiere además aplicar los conocimientos profesionales de los obtentores y evaluar el valor agronómico y comercial de las selecciones (UPOV, 2000a).

En la cuadragésima primera sesión del Comité, se señaló:

La importancia de que existiera una variación en el material existente en la naturaleza que sirviera de base de una variedad. Se da a entender que esta observación se apoya en el lenguaje del Artículo 6.1.a del Acta de 1978, cuya parte en cuestión establece:

"Sea cual sea el origen, artificial o natural, de la variación inicial que ha dado lugar a la variedad, ésta debe poder distinguirse claramente por uno o varios caracteres importantes de cualquier otra variedad, cuya existencia sea notoriamente conocida en el momento en que se solicite la protección".

Estas palabras respaldan la noción de que a fin de que se reproduzca, deberá existir una variación mínima, a partir de la que se haya producido la selección o selecciones. En caso de que no exista la selección a partir de la variación, a los fines del Convenio de la UPOV, se da a entender que no existe un descubrimiento susceptible de protección. En este contexto se indica, además, que la multiplicación o reproducción del material no modificado y seleccionado de entre la variación, así como su evaluación, constituye la puesta a punto a los fines del Acta de 1991 (UPOV, 2000, p. 4).

Al finalizar este Comité, se concluyó que hay quienes opinan que sólo se cumple con el criterio de puesta a punto si la propia planta descubierta se modifica luego de alguna manera y que 
la reproducción o multiplicación de la planta sin modificaciones genéticas no constituye una puesta a punto. Es decir, que para quienes sostienen dicha opinión es necesario que la planta descubierta se reproduzca en forma sexual y que se efectúe una selección en la progenie para tener una prueba de la puesta a punto. Sin embargo, para el Comité el enfoque antes seńalado no es correcto puesto que la selección en la progenie constituiría un fitomejoramiento, y con esta postura también se denegaría la protección a la mayoría de las mutaciones, puesto que la mutación, por lo general, se reproduce o multiplica sin modificaciones (UPOV, 2008, p. 6).

En lo referente a la puesta a punto, para Sánchez Castillo y Vanegas Avilés se considera como un mero hallazgo el proceso que incluye la observación de una variación natural de una especie vegetal, su identificación, aislamiento, selección, reproducción o multiplicación, caracterización y evaluación (2008, p. 340). Es decir, que, para estos autores, requiere algún tipo de proceso científico para lograr la nueva variedad.

El fitomejoramiento es imprescindible en varios sectores para lograr una buena seguridad alimentaria, un medio ambiente limpio, la sostenibilidad y varios procesos de transición en el ámbito rural. En el ámbito económico, el fitomejoramiento es un procedimiento que permitiría importantes ingresos para los países, a partir de su énfasis en los productos finales. Uno de los países que destaca en este sentido es Holanda, la cual tiene importantes ingresos de cultivos hortícolas, ornamentales, a partir del fitomejoramiento, investigación y fitogenética (UPOV, 2010, p. 6). El fitomejoramiento busca la innovación en el desarrollo y aplicación de nuevas tecnologías, el acceso a recursos genéticos y el capital para utilizar esos factores, ámbitos de gran importancia para el desarrollo de los países. De ahí que el acceso a la tecnología y al material genético sea el punto de partida para la puesta a punto de obtenciones vegetales. Todo esto nos permite afirmar la importancia del fitomejoramiento en términos de competitividad y rentabilidad en la cadena alimentaria (los agricultores y los obtentores tienen interés de fomentar la competencia del mercado de semillas).

En conclusión, la puesta a punto es un elemento vital para evitar la apropiación indebida de recursos naturales y de variedades vegetales sin fitomejoramiento. Ahora bien, esto trae ciertas dificultades pues se ha aceptado como tal ejecución el proceso de reproducción o multiplicación y evaluación de la variedad. La selección de mutantes naturales sí se permitiría y el proceso consistiría en la selección de la planta más fuerte, de determinadas características necesarias para el obtentor, posteriormente una propagación de la variedad, que en términos de la UPOV significaría reproducción o multiplicación, dependiendo del tipo de planta y su forma de reproducción. Lo que significaría dos cosas; la primera, que no existe una verdadera aplicación de una técnica o fitomejoramiento vegetal, de lado la puesta a punto de una variedad, más aún si nos encontramos ante una selección de mutantes naturales; la segunda, si se permite la multiplicación y reproducción como puesta a punto, se permite la protección de las variedades descubiertas, pues la multiplicación significaría un aumento en la cantidad, no una transformación genética. Para los métodos, que fueron antecedidos de un descubrimiento, se debería eliminar como forma de cumplimiento de la puesta a punto o mejoramiento la multiplicación o reproducción, pues supone la permisibilidad en la protección de los descubrimientos aumentados en cantidad.

\section{Implicaciones del Artículo 14.5. c) de la UPOV de 1991}

El Artículo 14.5. c) del Acta de la UPOV prevé la posibilidad de que el obtentor consiga su título sobre variedades esencialmente derivadas, mediante métodos como la selección de mutantes naturales, y señala que 
las variedades esencialmente derivadas podrán obtenerse, por ejemplo, por selección de un mutante natural o inducido o de una variante somoclonal, selección de un individuo variante entre las plantas de la variedad inicial, retrocruzamientos o transformaciones por ingeniería genética (Art. 145 c,) UPOV, 1991).

En las Actas de la UPOV de 1961 y de 1978 no se hacía referencia a los métodos de obtención, lo único que se señalaba era lo dispuesto en el Artículo 6 del Acta de 1978 (UPOV, 2010, p. 6) referente a la distinción. Sin embargo, en el Acta de 1991 se ampliaron los derechos del obtentor, incluyendo métodos y la distinción se ha llevados a otro apartado.

Cabe recalcar que Ecuador no es suscriptor del Acta de 1991, pero la Decisión No345 de la CAN ha tomado una gran cantidad de los principios de esa Acta incluyendo los derechos de los obtentores sobre variedades esencialmente derivadas. Aunque la CAN y el COESC no tienen un sistema claro de registro de variedades esencialmente derivadas, tampoco han excluido esa posibilidad. Por lo tanto, nuestra normativa no excluye ningún método, por lo que se podría pensar que están permitidos todos los que señala el Acta de 1991.

En el Artículo 14.5. c) se pueden identificar varios elementos, el primero de ellos es el uso del verbo "podrán", lo que significa que dichos métodos no tienen por qué originar necesariamente una variedad esencialmente derivada, sino que podría ser usado cualquier otro (UPOV, 2009). Si bien existen varios métodos que se ejemplifican en la norma (Acta de 1991 de la UPOV), el que resalta es el método: selección de variantes o mutantes. En los documentos de la UPOV no se excluye la posibilidad de que este método sea natural, lo que quiere decir que está permitido proteger variedades en las que no existe ningún tipo de intervención científica o técnica por parte del humano.

Este uso literal ha sido objeto de críticas, es así que el Comité de la UPOV señaló que algunos métodos de fitomejoramiento suscitaban inquietud porque se consideraban que generaban plagios; en particular, la selección de mutantes o el uso de retrocruzamientos repetidos para generar diferencias en caracteres de importancia menor a efectos del valor de la variedad. En el caso de las mutaciones, la preocupación es mayor para el sector de ornamentales, porque las diferencias pueden parecer visualmente grandes, pero se podría caer en un error, pues no necesariamente lo son. Se expusieron diversos argumentos para incluir en las directrices de examen de caracteres relaciones con el valor de la variedad — los denominados "caracteres importantes"-, con el fin de minimizar el riesgo de plagio. No obstante, en el debate se puso de manifiesto que la propuesta no era realmente adecuada, dado que la importancia de los caracteres puede variar en función del uso que se haga de una variedad, y puede evolucionar (Guiard, 2009, p. 10). De esta forma, la solución proporcionada por la UPOV es el examen DHE, que no soluciona el problema, sino que trata únicamente de la variabilidad, es decir, se refiere a las diferencias entre la variedad inicial y la variedad esencialmente derivada.

La norma expone algunos métodos de obtención, como parte de estos la "fecundación cruzada controlada de líneas parentales”, en el que la semilla resultante de la fecundación cruzada hereda su conformación genética de las líneas parentales. Esas variedades, denominadas híbridos, serán típicamente más vigorosas (presentarán "vigor híbrido") que las líneas parentales en las que se basan, dando origen, por ejemplo, a plantas de mayor rendimiento, mayor resistencia al estrés, etc. La misma fecundación cruzada controlada deberá repetirse cada vez que se produzca la semilla de esa variedad (Guiard, 2009, p. 10).

El siguiente método que la norma prevé, son las "mutaciones" que según la UPOV son un cambio abrupto en la estructura genética; es el resultado de un error en la transmisión de la información genética y puede compararse con un error en la transcripción de un texto. Una mutación produce una nueva variedad, por lo tanto, la búsqueda de mutaciones naturales 
(espontáneas) y la creación artificial de mutantes son una forma de producir nuevas variedades. Las mutaciones naturales son una fuente importante de variación y muchas variedades comercialmente importantes son mutantes (UPOV, 1996, p. 148). Como se puede ver, la norma permite que los descubrimientos sean protegidos, basta con el hecho de la naturaleza para ser considerado como registrable, un error que podría incluso poner en riesgo el patrimonio natural de nuestro país. Se debe reiterar que en Ecuador no se ha dispuesto nada y al parecer los métodos no estarían excluidos, dado que no existe una regulación que identifique los métodos permitidos. Pese a la justificación de la UPOV, que señala que las mutaciones son una fuente importante de variación y de importantes variedades, en nuestro país parecería que no tiene aplicabilidad esa justificación.

Otro de los métodos que la norma ha previsto, son las "variedades reproducidas por vía sexuada o los retrocruzamientos", esta consiste en cruzar la línea con una planta que presente un carácter diferente de herencia simple (por ejemplo, sedas que salen de la espiga roja y no amarillas), luego cruzar la descendencia con esta planta y repetir varias veces el cruzamiento de la descendencia con esta planta seleccionada para el carácter "sedas rojas". Al final, se obtiene una línea parecida a la inicial, que ha cambiado únicamente el color de la seda. Es posible también trabajar sobre los genes de una variedad que regulan la expresión de los caracteres cualitativos $y$, por medio, por ejemplo, de retrocruzamientos es posible modificar una buena variedad, obteniendo una que sea diferente, pero conservando inalterado la porción del genotipo de la variedad inicial.

Para la UPOV la condición de puesta a punto tiene excepciones, existe un grupo de partes que considera que no basta con la multiplicación y reproducción, como se ampliará a continuación. Según la UPOV, para la selección natural existe un trato especial en estos términos: Se exige que la planta descubierta se reproduzca en forma sexual y que se efectúe una selección de la progenie para tener prueba de la puesta a punto - Postura de contraria a la UPOV_- Este enfoque no puede ser correcto puesto que la selección en la progenie constituiría un "fitomejoramiento". Con este enfoque también se denegaría la protección a la mayoría de las mutaciones puesto que la mutación por lo general se reproduce o multiplica sin modificaciones -Postura UPOV-.

La UPOV, ante las diferentes posiciones que existen sobre los métodos de obtención de variedades esencialmente derivadas, propone a los países lo siguiente:

Se espera que las variedades comprendan "mejoras" que resulten beneficiosas para el público, pero la mejora en este sentido no es una condición para la concesión de la protección. Se ha sugerido que una variedad que sea descubierta (seleccionada) en la naturaleza y reproducida o multiplicada/evaluada/no modificada, no debería ser susceptible de protección, dado la naturaleza "de connotación política de la variación inicial a partir de la que ha sido puesta a punto" (UPOV, 2000b).

Sin embargo, posteriormente a esta recomendación, en la Cuadragésima primera sesión del Comité, el Presidente del Comité Técnico subrayó la importancia de que existiera una variación en el material existente en la naturaleza que sirviera de base de una variedad, en los siguientes términos:

Sea cual sea el origen, artificial o natural, de la variación inicial que ha dado lugar a la variedad, ésta debe poder distinguirse claramente por uno o varios caracteres importantes de cualquier otra variedad, cuya existencia sea notoriamente conocida en el momento que se solicite la protección (UPOV, 2000b). 
Esto quiere decir que, aún si estamos frente a un descubrimiento debe existir una variación mínima a partir de la que se haya producido la selección, más aún la norma señala que no existen descubrimientos sujetos a protección.

Según la Association for Plant Breeding for the Benefit of Society (APBREBES), en las Declaraciones sobre los borradores de las Notas Explicativas en la Agenda del CAJ-AG 9, 14/10/2014, señalan que

Es comprensible que una mutación que se encontró en huerto frutal de agricultores o en una planta ornamental cree controversias sobre quién es el titular de los derechos. Para el titular de los derechos de la variedad inicial, podría significar un nuevo título de derechos para la misma inversión, con otro período completo de 20 o 25 años.

Para la persona que encontró la mutación, podría significar un período completo de licencia, sin haber invertido en el trabajo de mejoramiento o sin haber comprado el título.

Por lo tanto, las mutaciones deberían ser excluidos de protección. Esta trae dudas sobre el propósito de la Convención de la UPOV. Debe sustituirse y anotar que las mutaciones naturales no son sujetas de protección. O por lo menos la norma solo debe mencionar mutaciones inducidas, pero no mutaciones naturales. Esto tiene mucha importancia aun cuando los métodos son solo ejemplificativos, por tanto, debe suprimirse la selección de mutantes naturales (Gura, 2014).

El Acta de la UPOV ha establecido estos métodos con el fin de ejemplificar que, como se ha visto, existen métodos en los que se puede verificar una real aplicación de una técnica; sin embargo, en el caso especial de la selección de mutantes naturales no se puede identificar esa aplicación de fitomejoramiento, lo que genera varias dudas. Primero, la problemática se basa esencialmente en la falta de aplicación de una técnica para obtener una variedad y este método permitiría muchos registros sin haber "creado" una variedad, tanto para obtentores como agricultores. Segundo, la UPOV no tiene una posición clara sobre si permitir o no la protección de las variedades esencialmente derivadas obtenidas mediante el método, es decir, la selección de un mutante natural. En los informes se puede ver que las veces que se ha aceptado este método enfáticamente se justifican en que, si no se aceptarán las mutaciones naturales, se estaría perdiendo la posibilidad de desarrollar un gran número de variedades con un potencial valioso para la sociedad. Posiciones contrarias a ésta afirman que no se debe permitir la protección de variedades espontaneas, más se debe verificar la puesta a punto.

Tercero, para concluir cabe recalcar que en Ecuador no existe un sistema claro de protección de variedades esencialmente derivadas, simplemente se las nombra en un solo artículo, dejando entrever que el sistema excluye las posibilidades de registro de esta categoría. En el Artículo 466 del COESC se hace referencia a esta categoría de obtenciones vegetales, pero únicamente referente a las excepciones del obtentor. Por otra parte, la Decisión No345, como se ha señalado anteriormente, tiene mayor jerarquía y se exponen los conceptos con mayor amplitud, específicamente, en el Artículo 3, donde se aportan sus definiciones. Y, posteriormente, los Artículo 24 y 25 establecen las limitaciones de uso del material de variedades esencialmente derivadas.

Como consecuencia de lo señalado anteriormente, y de la ambigüedad de las normas con respecto a las variedades esencialmente derivadas, se podría concluir que: 1) El COESC ha optado porque todas las obtenciones vegetales, sin importar el método de obtención utilizado y sin mayor análisis de aplicación de técnicas, se postulen como nuevas variedades. Posiblemente las dificultades de distinguir entre una variedad inicial y una variedad esencialmente derivada residen en que se necesita una metodología de investigación y análisis que requeriría mucha tecnología y conocimiento de biogenética para poder determinar si las modificaciones en la 
progenie son suficientes para poder considerarla como una nueva variedad. 2) El COESC, a diferencia de la Ley de Propiedad Intelectual vigente hasta el año 2016, se apegaba mucho más a los conceptos del Acta de la UPOV de 1991. Aun siendo suscriptores del Acta de 1978, con las modificaciones que se hicieron a la normativa de Propiedad Intelectual, el COESC parecería apegarse mucho más al Acta de 1978 dejando de lado la posibilidad de otorgar certificado de obtentor de variedades esencialmente derivadas. Sin embargo, vale recordar que el concepto se encuentra presente en la norma, pero sin mayores referencias. 3) El hecho de que todas las obtenciones vegetales se registren como variedades iniciales podría permitir que cualquier método de obtención sea utilizado y se otorguen todos los derechos, permitiendo así tener una cadena infinita de mutaciones sobre una misma variedad que podría ser objeto de protección de una variedad inicial.

\section{Referencias bibliográficas}

Canaval, J. (2008). Manual de Propiedad Intelectual. Rosario: Universidad de Rosario.

Cervantes, M. (2015). Hibridaciones en plantas hortícolas; mejora vegetal. Escuela Familiar Agraria. <http://www.infoagro.com/hortalizas/hibridaciones_horticolas.htm>.

Consejo Argentino para la Información y el Desarrollo de la Biotecnología (2015). Diversidad, Selección Natural y Selección Artificial. El Cuaderno, 39. <http://www.porquebiotecnologia.com.ar/index.php?action=cuaderno\&opt=5\&tipo=1\&note=39>.

Fernández Ruiz, P. L. Hibridación de los ácidos nucleicos. Fundamento teórico. <http:// www.seap.es/c/document_library/get_file?uuid=dd48aeb8-9e38-4541-bcfbd75fd6b2ee9f $>$.

Guiard, J. (2009). La Redacción en las Disposiciones sobre Variedades Esencialmente Derivadas. Ginebra: UPOV.

Gura, S. (2014). Statements and Drafts of Explanatory Notes on the Agenda of CAJ-AG9, $14 O c$ tober 2014. Bonn: UPOV.

Idareta Olagüe, E. (2004). Estudio de caracteres productivos de hibridos de Pleurotus ostreatus en condiciones semi-industriales para su selección dentro de un programa de mejora genética. Navarra: Universidad Pública de Navarra.

INFO AGRO (2014). INFOAGRO. <http://www.infoagro.com/hortalizas/hibridaciones_horticolas.htm>.

Krieger, E. (2013). Puntos de Vista de la Comunidad Internacional de Obtentores de Plantas Ornamentales y Frutales de Reproducción Asexuada (CIOPORA) respecto a las Variedades Esencialmente Derivadas. Ginebra: UPOV.

Muñoz, F. (2002). Plantas medicinales y aromáticas. Madrid: Grupo Mundi-Prensa.

Patiño, M. (1998). Los Derechos de los Obtentores de Nuevas Variedades Vegetales. Bogotá: Ediciones Jurídicas Gustavo Ibañez Ltda.

Sánchez Castillo, C. y Vanegas Avilés, L. M. (2008). Las Obtenciones Vegetales y los Derechos de Propiedad Intelectual en Costa Rica. Ciencias Económicas, 26 (1), 335-347.

UNNE (2015). Reproducción Asexual (con énfasis en el reino Planta). Hipertextos del Área de la Biología. <http://www.biologia.edu.ar/reproduccion/asexual.htm>.

\section{Legislación}

Asamblea Nacional de la República de Ecuador (2016). Código Orgánico de la Economía Social de los Conocimientos, Creatividad e Innovación (COESC). 19 de noviembre de 2016. 
Comunidad Andina (1993). Decisión N³45 sobre el Régimen Común de Protección a los Derechos de los Obtentores de Variedades Vegetales. Aprobado por la Comisión del Acuerdo de Cartagena el 21 de octubre de 1993.

Congreso Nacional de la República del Ecuador (2006). Ley de propiedad intelectual. 28 de diciembre de 2006.

\section{Convenciones, informes y sesiones}

Unión internacional para la protección de obtenciones vegetales, UPOV (2010). Informes de Representantes de Miembros y Observadores sobre los Ámbitos Legislativo, Administrativo y Técnico Cuadragésima cuarta sesión ordinaria. 21 de octubre 2010.

- (2009). Notas Explicativas sobre las Variedades Esencialmente Derivadas con Arreglo al Acta de 1991 del Convenio de la UPOV, 22 de octubre del 2009.

- (2008). Quincuagésima octava sesión del Comité Administrativo y Jurídico/ Ginebra, 27 y 28 de octubre de 2008.

- (2002). La Noción del Obtentor y lo Notoriamente Conocido en el Sitema de Protección Vegetal basado en el Convenio de la UPOV. Aprobado por el Consejo de la UPOV en su decimonovena sesión extraordinaria, 19 de abril del 2002

- (2000a). Informe aprobado por el Comité Administrativo y Jurídico de la cuadragésima primera sesión de Ginebra, 6 de abril de 2000.

- (2000b). La Noción de Obtentor y de lo Notoriamente Conocido, Comité Administrativo y Jurídicol Cuadragésima segunda sesión, 23 y 24 de octubre de 2000.

- (1996). Ley tipo sobre la Protección de las Obtenciones Vegetales (Publicación No. 842). Ginebra: UPOV.

- (1991). Convenio Internacional para la Protección de las Obtenciones Vegetales, Acta 1991, 19 de marzo de 1991.

- (1978). Convenio Internacional para la Protección de las Obtenciones Vegetales, Acta 1978, 23 de octubre de 1978. 